\title{
The Effect of Pulsed Electromagnetic Fields on Microleakage of Amalgam Restorations: An in Vitro Study
}

\author{
Maryam Paknahad, ${ }^{1,2}$ Shoaleh Shahidi, ${ }^{1,3}$ Seyed Mohammad Javad Mortazavi, ${ }^{4,5}$ Ghazal Mortazavi, ${ }^{4}$ \\ Mahdi Saeedi Moghadam, ${ }^{4}$ and Ali Dehghani Nazhvani ${ }^{3,6,}$ \\ ${ }^{1}$ Prevention of Oral and Dental Disease Research Center, School of Dentistry, Shiraz University of Medical Sciences, Shiraz, IR Iran \\ ${ }^{2}$ Department of Oral and Maxillofacial Radiology, School of Dentistry, Shiraz University of Medical Sciences, Shiraz, IR Iran \\ ${ }^{3}$ Biomaterial Research Center, School of Dentistry, Shiraz University of Medical Sciences, Shiraz, IR Iran \\ ${ }^{4}$ Ionizing and Non-Ionizing Radiation Protection Research Center (INIRPRC), Shiraz University of Medical Sciences, Shiraz, IR Iran \\ ${ }^{5}$ Medical Physics and Medical Engineering Department, School of Medicine, Shiraz University of Medical Sciences, Shiraz, IR Iran \\ ${ }^{6}$ Oral and Maxillofacial Pathology Department, School of Dentistry, Shiraz University of Medical Sciences, Shiraz, IR Iran \\ "Corresponding author: Ali Dehghani Nazhvani, Oral and Maxillofacial Pathology Department, Biomaterial Research Center, School of Dentistry, Shiraz University of Medical \\ Sciences, Shiraz, IR Iran. Tel: +98-989177160991, E-mail: alidehghaninazhvani@yahoo.com
}

Received 2015 August 11; Revised 2015 December 26; Accepted 2016 January 03.

\begin{abstract}
Background: Previous studies have reported an increase in the mercury release from dental amalgam restorations, following exposure to electromagnetic fields generated by sources such as mobile phones and magnetic resonance imaging (MRI). It has also been shown that MRI increases microleakage of amalgam restorations. In this study, Helmholtz coils are used for generating pulsed electromagnetic fields (PEMF).

Objectives: The purpose of this study was to evaluate the effect of PEMFs on microleakage of amalgam restorations, using a pair of Helmholtz coils.

Patients and Methods: Standardized class V cavities were prepared on the facial surfaces of 46 non-carious extracted human premolars. Then, the samples were randomly divided into experimental and control groups, each containing 23 teeth. The experimental group was exposed to the uniform magnetic fields generated by a pair helmholtz coils. The magnetic field strength at the central point of the two coils was $0.1 \mathrm{mT}$. All specimens were placed in $2 \%$ basic fuchsin solution. Then the teeth were sectioned, examined under a stereomicroscope, and scored for microleakage according to the degree of dye penetration.

Results: There was no significant difference between the two groups regarding the microleakage score.

Conclusions: The results of the present study suggest that PEMF exposure does not have adverse effects on microleakage of amalgam restorations.
\end{abstract}

Keywords: Pulsed Electromagnetic Fields, Amalgam, Microleakage, Helmholtz Coils

\section{Background}

Magnetic fields can be demonstrated by lines of force and these fields are produced by electric current flow. Electromagnetic fields can be either pulsed or static. Static magnetic fields (SMF) are formed around a permanent magnet or by direct current (DC) flow, while time-varying magnetic fields are produced by alternating currents (AC) with a frequency above zero $(1,2)$. Human beings are daily exposed to natural and artificial electromagnetic fields (EMF) originating from various sources (3). According to the world health organization(WHO), factors such as growing electricity demands, ever-advancing technologies and changes in social behavior result in increasing exposure to man-made EMFs (4). The rapidly increasing growth of the human exposure to EMFs has led to growing concern about its possible adverse health effects. EMFs can be produced by all electrical or electronic devices, such as wireless tech- nologies (e.g. Wi-Fi, mobile phones and cordless phones), laptop computers, microwave ovens and power lines (5). Helmholtz coils are devices that generate pulsed electromagnetic fields (PEMF).

Helmholtz coils are named in honor of Hermann von Helmholtz, the German physicist (6). This device consists of two circular coils of wire with identical electrical currents which flow in the same direction. In a pair of Helmholtz coils, with their two current loops, each with $\mathrm{N}$ turns and radius $\mathrm{R}$, which are separated by a distance $\mathrm{R}$, the magnetic field at any point along the axis of the coils (z) can be calculated by summing the individual magnetic fields of the coils using the superposition principle. In this configuration, Biot-Savart law is used to measure the magnetic field at the center of this system when $\mathrm{z}=0$ as follows:

$B(z=0)=\frac{8}{5 \sqrt{5}} \times \frac{\mu_{0} \mathrm{IN}}{R}$ 
Where: $\mathrm{B}$ is the magnitude of the magnetic field, $\mathrm{R}$ is the radius of the loop, $\mathrm{N}$ is the number of turns in the current loop., $\mu_{0}$ is the permeability of free space, I is current.

Over the past years, our team has investigated the effects of exposure to different common and or occupational sources of EMFs, such as cellular phones (7-14), mobile base stations (15), mobile phone jammers (16), laptop computers (17), radars (8), dentistry cavitrons (18) and MRI (13, 19). The findings of our studies have clearly shown that exposure to magnetic fields in MRI or microwave radiation, emitted from mobile phones, significantly increases the mercury release from dental amalgam restorations (13, 19). Furthermore, new studies have shown that X-ray, as a part of the electromagnetic spectrum, can increase the mercury release from amalgam fillings (20). Microleakage has been suggested to be a significant problem leading to pulp pathology, post-operative pain, tooth discoloration, recurrent caries and accelerated deterioration of the fillings $(21,22)$. Previous studies have shown that EMFs emitted in MRI increase the microleakage of amalgam restorations $(23,24)$. Shahidi et al. suggested that the main effect of the strong magnetic fields was development of thermoelectromagnetic convection, which is responsible for increased diffusion, grain boundary migration, and vacancy formation, resulting in microleakage (23). However, the magnitude of temperature increase in MRI is not high enough to justify this theory. The accelerated corrosion due to galvanic effect may explain the increased microleakage of amalgam following EMFs exposure. To the best of our knowledge, this is the first study performed on the effect of PEMFs on amalgam microleakage.

\section{Objectives}

Therefore, the null hypothesis of this study is if exposure to PEMFs generated by a designed Helmholtz Coil increases the microleakage of amalgam.

\section{Patients and Methods}

\subsection{Teeth Samples}

The present study was approved by the ethics committee of Shiraz University of Medical Sciences. Forty six noncarious, recently extracted premolar and molar teeth, were selected for this study. The teeth were stored in saline solution for up to 2 months after cleaning and surface debridement. The teeth with fractures or structural defects were excluded. Standardized class V cavities ( $3 \mathrm{~mm}$ length, $5 \mathrm{~mm}$ wide, $2 \mathrm{~mm}$ deep) were prepared on the buccal surface at the cementoenamel junction using carbide burs (SS White
Burs, Lakewood, NJ) and in a high speed turbine with airwater spray using a template. A separate bur was used after every 6 cavity preparations to ensure cutting efficiency. The cavities were restored with Cinalux amalgam (nongama-2, spherical amalgam, Faghihi Dental, Tehran, Iran). The amalgams were triturated according to manufacturers' directions, and then they were condensed incrementally towards the cavity walls using small condensers. All the procedures for restoration of the cavities including cavity preparation, burnishing and polishing were performed by the same clinician. The restored teeth were placed in saline solution at $37^{\circ} \mathrm{C}$ for seven days. The teeth were randomly divided into two groups each containing 23 teeth.

\subsection{Exposure of the Samples}

Two pairs of identical Helmholtz coils were designed and manufactured in the ionizing and non-ionizing radiation protection research center (INIRPRC) at Shiraz University of Medical Sciences, Shiraz, Iran. The device was designed in our previous study (1). The basic characteristics of the coils used in this study have been explained in another paper $(1,5)$. These coils with 100 turns in each loop were framed on Teflon. The coils were spaced apart at a distance equal to their radii and the distance between the coils was $12.5 \mathrm{~cm}$. The outer diameter of the coils was 13.5 $\mathrm{cm}$. The coils were driven by a sinusoidal signal from a function generator (GFG-8020H, GW Instrument Co., Ltd). A recently calibrated Gauss meter (Lutron 828, Taiwan) was used for measuring the magnetic field (B) at the central point between the coils. In the central area of the coils, the magnetic field was relatively uniform (with alterations less than 5\%). In this study, the earth magnetic field was not shielded.

\subsection{Microleakage Evaluation}

Two layers of nail varnish were applied on the entire teeth surfaces except for the restorations and $1 \mathrm{~mm}$ around them. The specimens were immersed in $2 \%$ basic fuchsin dye solution (merck, Germany) at the room temperature for 24 hours and then they were rinsed in tap water and dried. A slow speed water- cooled saw was used to section each tooth buccolingually. The section corresponding to the central portion of the tooth restoration was examined at the gingival, axial and occlusal margins under a stereomicroscope (Olympus. Tokyo, Japan) at $80 \times$ magnification by the examiner who was blinded to the groups. The degree of microleakage was evaluated according to a standard ranking in which $0=$ No dye penetration; $1=$ Dye penetration along the enamel; 2 = Dye penetration along the dentine-enamel junction (DEJ) but not including the axial wall and $3=$ Dye penetration along the axial wall (6). 
The data were analyzed using the Mann-Whitney U-test to compare microleakage in the case and control groups to identify any statistically significant differences at the significance level of $0.95(\mathrm{P}<0.05)$.

\section{Results}

The distribution of the scores of microleakage in each group is presented in Table 1 . The scores of microleakage showed no significant difference between the exposure and control groups $(\mathrm{P}=0.345)$. The percentage of the teeth with grade 3 in the exposed group was $8.6 \%$, while in the control group it was zero. The percentage of the teeth with no dye penetration (grade1) in the exposed group and in the control group was approximately the same.

\section{Discussion}

Increase in the microleakage of dental amalgam restorations following exposure to electromagnetic fields of MRI has been reported in previous studies $(23,24)$. Electromagnetic fields can be either pulsed or static. In the present study, we found that PEMF exposure generated by Helmholtz coil does not have adverse effects on microleakage of amalgam restorations.

Dental amalgam has been one of the oldest filling materials in posterior teeth restorations because of its several advantages, such as easy manipulation, low technique sensitivity, high wear resistance, low cost, durability and insolubility in oral fluids $(21,25,26)$. However, one of the disadvantages of dental amalgam is the lack of chemical adhesion to the tooth structure which can result in amalgam microleakage. Microleakage is defined as the clinically undetectable penetration of fluids, ions or molecules from the oral cavity through the gaps between the cavity walls and the restoration material. Microleakage prevention is crucial for longevity of restorations since the passage of bacteria can lead to recurrent carries and pulpal inflammation which may cause reversible pulpitis or tooth necrosis. The tooth at last may need a replacement restoration, endodontic or even extraction (21). Different methods have been used for the evaluation of dental amalgam restorations microleakage. Among them, dye penetration test is a simple, inexpensive and qualitative method (21). Therefore, we used this technique for the assessment of microleakage.

In the present study, two layers of varnish coating were applied around restoration margins and the pulpal surfaces as a barrier to decrease microleakage around amalgam restoration and prevent undesirable dye penetration. Varnish, acting as a mechanical barrier, did not bond to the tooth structure or amalgam restoration. However, other adhesive liners, such as resin based liners, can decrease the microleakage of amalgam restorations and affect the results of the study $(21,27)$.

In the present study, it was revealed that amalgam microleakage was not significantly different in the PEMF exposed group compared to the control group. This finding was in contrast with the results obtained in our previous studies carried out on the increased release of mercury from amalgam restorations after being exposed to different sources of EMFs, such as MRI or radiations emitted by mobile phones (13). There are few studies which have evaluated the effects of magnetic fields in MRI on microleakage of amalgam restorations $(23,24,28)$. Some studies have suggested that the magnetic field of MRI increase microleakage of amalgam restorations $(23,24)$. Shahidi et al. have suggested that the increase in microleakage following MRI might be attributed to the thermoelectromagnetic convection induced by exposure to EMFs that was supposed to be responsible for the enhancement of the diffusion process, grain boundary migration and vacancy formation resulting in microleakage (23). However, Mortazavi and Paknahad believed that the magnitude of temperature increase is not high enough to justify this theory (29). On the other hand, Akgun et al. did not indicate any statistically significant differences in the extent of microleakage in groups with or without MRI exposure (28).

The present study was an in vitro test. One of the limitations of in vitro tests is that they do not simulate clinical conditions precisely. Therefore, the results of these tests should be applied to the clinical situation only after being substantiated by in vivo evidence requiring long-term clinical studies. Thus, further investigations are highly recommended.

\subsection{Conclusion}

In conclusion, owing to the controversy existing over the potential adverse effects of dental amalgams, the replacement of amalgam with other restorative materials does not seem well-grounded. Therefore, considering the significance of this challenging issue, further research is necessary to verify the effects of different sources of EMFs on amalgam restorations.

\section{Acknowledgments}

The authors thank the Vice-Chancellery of Shiraz University of Medical Sciences for supporting this research (Grant \# 7994); Dr. Vosoughi for statistical analysis and Dr. Amalsaleh for English editing. 
Table 1. The Distribution of the Scores in the Control and PEMF Exposure Groups

\begin{tabular}{|c|c|c|c|c|}
\hline \multirow[b]{2}{*}{ Grade } & \multicolumn{4}{|c|}{ Percent of the Scores } \\
\hline & 0 & 1 & 2 & 3 \\
\hline Control group & 82.6 & 13 & 4.3 & 0 \\
\hline PEMF exposure group & 73.9 & 4.3 & 13 & 8.6 \\
\hline
\end{tabular}

\section{Footnote}

Funding/Support: This study was supported by the biomaterial research center, school of dentistry and ionizing and non-ionizing radiation protection research center(INIRPRC), Shiraz University of Medical Sciences (SUMS), Shiraz, Iran.

\section{References}

1. Haghnegahdar A, Khosrovpanah H, Andisheh-Tadbir A, Mortazavi G, Saeedi Moghadam M, Mortazavi S, et al. Design and fabrication of helmholtz coils to study the effects of pulsed electromagnetic fields on the healing process in periodontitis: preliminary animal results. $J$ Biomed Phys Eng. 2014;4(3):83-90. [PubMed: 25505775].

2. Nursal TZ, Bal N, Anarat R, Colakoglu T, Noyan T, Moray G, et al. Effects of a static magnetic field on wound healing: results in experimental rat colon anastomoses. Am J Surg. 2006;192(1):76-81. doi: 10.1016/j.amjsurg.2006.01.024. [PubMed:16769280].

3. Yamaguchi-Sekino S, Sekino M, Ueno S. Biological effects of electromagnetic fields and recently updated safety guidelines for strong static magnetic fields. Magn Reson Med Sci. 2011;10(1):1-10. [PubMed: 21441722].

4. WHO . Electromagnetic fields(EMF):What are electromacnetic fields? 2015. Available from: Available from: http://www.who.int/peh-emf/ about/WhatisEMF/en/.

5. Mortazavi G, Haghani M, Rastegarian N, Zarei S, Mortazavi SMJ. Increased release of mercury from dental amalgam fillings due to maternal exposure to electromagnetic fields as a possible mechanism for the high rates of autism in the offspring: introducing a hypothesis. Biomed Phys Engin J. 2015.

6. Wikipedia. .Helmholtz coil. 2015.

7. Mortazavi SM, Motamedifar M, Namdari G, Taheri M, Mortazavi $\mathrm{AR}$, Shokrpour N. Non-linear adaptive phenomena which decrease the risk of infection after pre-exposure to radiofrequency radiation. Dose Response. 2014;12(2):233-45. doi: 10.2203/dose-response.12055.Mortazavi. [PubMed: 24910582].

8. Mortazavi SM, Taeb S, Dehghan N. Alterations of visual reaction time and short term memory in military radar personnel. Iran J Public Health. 2013;42(4):428-35. [PubMed: 23785684].

9. Mortazavi SM, Rouintan MS, Taeb S, Dehghan N, Ghaffarpanah AA, Sadeghi Z, et al. Human short-term exposure to electromagnetic fields emitted by mobile phones decreases computer-assisted visual reaction time. Acta Neurol Belg. 2012;112(2):171-5. doi: 10.1007/s13760012-0044-y. [PubMed: 22426673].

10. Mortazavi S, Mosleh-Shirazi M, Tavassoli A, Taheri M, Mehdizadeh A, Namazi S, et al. Increased Radioresistance to Lethal Doses of Gamma Rays in Mice and Rats after Exposure to Microwave Radiation Emitted by a GSM Mobile Phone Simulator. Dose Response. 2013;11(2):28192. doi: 10.2203/dose-response.12-010.Mortazavi. [PubMed: 23930107].

11. Mortazavi SMJ, Mosleh-Shirazi M, Tavassoli A, Taheri M, Bagheri Z, Ghalandari R, et al. A comparative study on the increased radioresistance to lethal doses of gamma rays after exposure to microwave radiation and oral intake of flaxseed oil. Iranian J Rad Res. 2011;9(1):9-14.

12. Mortazavi SMJ, Habib A, Ganj-Karimi AH, Samimi-Doost R, Pour-Abedi A, Babaie A. Alterations in TSH and thyroid hormones following mobile phone use. Iranian J Med Sci. 2015;34(4):299-300.

13. Mortazavi SM, Daiee E, Yazdi A, Khiabani K, Kavousi A, Vazirinejad R, et al. Mercury release from dental amalgam restorations after magnetic resonance imaging and following mobile phone use. Pak J Biol Sci. 2008;11(8):1142-6. [PubMed: 18819554].

14. Mortazavi SM, Ahmadi J, Shariati M. Prevalence of subjective poor health symptoms associated with exposure to electromagnetic fields among university students. Bioelectromagnetics. 2007;28(4):326-30. doi: 10.1002/bem.20305. [PubMed: 17330851].

15. Mortazavi SMJ. Safety issue of mobile phone base stations. J Biomed Phys Eng. 2013;3(1):1-2.

16. Mortazavi SMJ. Adaptive responses after exposure to cosmic and natural terrestrial radiation. 2004

17. Mortazavi SMJ, Tavassoli A, Ranjbari F, Moammaiee P. Effects of laptop computers' electromagnetic field on sperm quality. Journal of Reproduction \& Infertility. 2010;11(4).

18. Mortazavi SM, Vazife-Doost S, Yaghooti M, Mehdizadeh S, RajaieFar A. Occupational exposure of dentists to electromagnetic fields produced by magnetostrictive cavitrons alters the serum cortisol level. J Nat Sci Biol Med. 2012;3(1):60-4. doi: 10.4103/0976-9668.95958. [PubMed: 22690053]

19. Mortazavi SMJ, Neghab M, Anoosheh SMH, Bahaeddini N, Mortazavi G, Neghab P, et al. High-field MRI and mercury release from dental amalgam fillings. Int J Occup Environ Med. 2014;5(2):101-5.

20. Kursun S, Oztas B, Atas H, Tastekin M. Effects of X-rays and magnetic resonance imaging on mercury release from dental amalgam into artificial saliva. Oral Radiol. 2014;30(2):142-6.

21. Bembi S, Bembi NN, Sood A, Gambhir A. To Evaluate the Effect of Different Adhesive Materials on the Microleakage of Bonded Amalgam Restorations: An in vitro Study. Int J Clin Pediatr Dent. 2012;5(3):185-9. doi: 10.5005/jp-journals-10005-1163. [PubMed: 25206165].

22. Morrow LA, Wilson NH, Setcos JC, Watts DC. Microleakage of amalgam cavity treatment systems: an in vitro evaluation. Am J Dent. 2002;15(4):262-7. [PubMed: 12572646].

23. Shahidi SH, Bronoosh P, Alavi AA, Zamiri B, Sadeghi AR, Bagheri $\mathrm{MH}$, et al. Effect of magnetic resonance imaging on microleakage of amalgam restorations: an in vitro study. Dentomaxillofac Radiol. 2009;38(7):470-4. doi: 10.1259/dmfr/30077669. [PubMed: 19767518].

24. Yilmaz S, Misirlioglu M. The effect of 3 T MRI on microleakage of amalgam restorations. Dentomaxillofac Radiol. 2013;42(8):20130072. doi: 10.1259/dmfr.20130072. [PubMed: 23674614].

25. Cenci MS, Piva E, Potrich F, Formolo E, Demarco FF, Powers JM. Microleakage in bonded amalgam restorations using different adhesive materials. Braz Dent J. 2004;15(1):13-8. doi: /S010364402004000100003. [PubMed: 15322639].

26. Mitchell RJ, Koike M, Okabe T. Posterior amalgam restorations-usage, regulation, and longevity. Dent Clin North Am. 2007;51(3):573-89. doi: 10.1016/j.cden.2007.04.004. [PubMed: 17586144] v.

27. Ben-Amar A, Cardash HS, Judes H. The sealing of the tooth/amalgam interface by corrosion products. J Oral Rehabil. 1995;22(2):101-4. [PubMed: 7722740]. 
28. Akgun OM, Polat GG, Turan Illca A, Yildirim C, Demir P, Basak F. Does magnetic resonance imaging affect the microleakage of amalgam restorations?. Iran J Radiol. 2014;11(3):eee15565. doi: 10.5812/iranjradiol.15565. [PubMed: 25763074].
29. Mortazavi S, Paknahad M, et al. Effect of magnetic resonance imaging on microleakage of amalgam restorations: an in vitro study. Dentomaxillofac Radiol. 2016;45(1):20150187. doi: 10.1259/dmfr.20150187. [PubMed: 26224142] Epub 2015 Aug 17. 